\title{
Special section on recent progress in numerical verification
}

Mathematical analysis of nonlinear problems encounters various difficulties. Thus, computer assisted proofs for nonlinear problems have attracted attention of many researchers. Especially, numerical computations with result verification is shown to be quite useful for such computer assisted proofs.

The special section focuses on this topic. This section starts with two invited papers. One is focused on computer assisted proofs in the research area of nonlinear partial differential equations. Another paper is a survey paper on robust algorithms in computational geometry. These two papers demonstrate usefulness of numerical computations with result verification both for scientific problems and engineering problems. After these papers, the following variety of topics is presented: numerical verification of chaos-based random number generators, interval multiple-precision arithmetic, a model problem for global optimization, computer assisted proof for nonlinear two-point boundary value problems, an enclosure method for complex eigenvalues and a computer-assisted stability proof for the Orr-Sommerfeld problem. The guest editors believe that this special section invites many readers to the frontier of this emerging field.

They would like to express their sincere thanks to all authors for their contributions. They also thank the reviewers and the members of the guest editorial committee, especially Prof. Tibor Csendes (University of Szeged), Prof. Philippe Langlois (University of Perpignan), Prof. Kaori Nagatou (Kyushu University), Prof. Takeshi Ogita (Tokyo Woman's Christian University), Prof. Katsuhisa Ozaki (Shibaura Institute of Technology), and the editorial staff of NOLTA Journal for their supports on publishing this Special Section.

\author{
Shin'ichi Oishi \\ Michael Plum \\ Siegfried M. Rump \\ Guest Editors
}

\section{Editorial Committee of the Special Section}

\section{Guest Editors:}

Shin'ichi Oishi (Waseda University), Michael Plum (University of Karlsruhe), Siegfried M. Rump (Hamburg University of Technology)

\section{Guest Associate Editors:}

Tibor Csendes (University of Szeged), Philippe Langlois (University of Perpignan), Kaori Nagatou (Kyushu University), Takeshi Ogita (Tokyo Woman's Christian University)

\section{Secretary of the Special Section:}

Katsuhisa Ozaki (Shibaura Institute of Technology) 